\title{
纵向数据半参数建模中的迭代加权 偏样条最小二乘估计
}

\author{
孙孝前
}

(淮阴师范学院数学系, 淮安 223001 ; 密苏里大学统计系, MO 65201, 美国)

\section{尤进红}

(Regina 大学数学与统计系, S4S 0A2, 加拿大)

\begin{abstract}
摘要考虑了纵向数据半参数建模中的估计问题, 提出了参数分量的一个迭代加权 偏样条最小二乘估计. 在渐近方差意义下该估计比加权偏样条最小二乘估计更加有 效, 且具有渐近正态性。另外, 给出了一个自适应方法, 该方法能保证经过有限次迭 代后，迭代过程会终止，并且产生的估计渐近等价于使用迭代方法所能产生的最好的 估计, 这些结果是 Chen 和 Shao 的结果在半参数回归上的推广.
\end{abstract}

\section{关键词 半参数建模 纵向数据 迭代加权偏样条最小二乘估计 渐近正态性}

在许多纵向数据的研究中, 人们通常希望建立输出变量和协变量及时间效应的关系. 一个 常见的困难是时间效应通常十分复杂以致难以进行参数化建模. 例如对生物生长、HIV 和荷 尔蒙的研究 ${ }^{[1 \sim 3]}$, 因此很多学者把注意力转移到半参数回归模型上. 在半参数回归模型中假定 有两部分的效应: 参数的协变量效应和非参数的时间效应. 一个重要的半参数回归模型是如下 定义的部分线性回归模型:

$$
y_{i j}=x_{i j}^{\prime} \beta+g\left(t_{i j}\right)+\varepsilon_{i j}, \quad i=1, \cdots, k, j=1, \cdots, n_{i}, \quad \sum_{i=1}^{k} n_{i}=n,
$$

其中 $y_{i j}$ 是第 $i$ 个个体在时间 $t_{i j}$ 的响应, $\beta=\left(\beta_{1}, \cdots, \beta_{p}\right)^{\prime}$ 是相应于个体水平协变量 $x_{i j}$ 的未 知参数向量, $g(\cdot)$ 是 $\mathbb{R}$ 上的未知函数, $\varepsilon_{i j}$ 是均值为 0 方差为 $\sigma_{i}^{2}$ 的随机误差, $\sigma_{i}^{2}$ 未知并且 不必相等; 此外对于 $j_{1}, j_{2}=1, \cdots, n_{i}, \varepsilon_{i j_{1}}$ 和 $\varepsilon_{i j_{2}}$ 亦可能相关.

当 $\left\{\varepsilon_{i j}\right\}$ 为平稳 Gauss 过程时, Zeger 与 Diggle ${ }^{[2]}$ 讨论了模型 $(0.1)$ 中 $\beta$ 和 $g(\cdot)$ 的估计问 题, 通过后移算法提出了一个半参数方法, 同时还把此方法应用到 HIV 研究中的 CD4 细胞数 的时间趋势的估计问题中. 当 $n_{i} \equiv 1$ 时, 模型 $(0.1)$ 退化为非纵向的部分线性回归模型. 关于 非纵向的部分线性回归模型估计和应用的综述可见文献 [4].

对于模型 (0.1), 在假定当 $k \rightarrow \infty$ 时 $n_{i}$ 也趋向于 $\infty$ 和没有组间相关性的条件下, Liang 
和 $\mathrm{Härdle}^{1)}$ 提出了 $\beta$ 的一个加权半参数最小二乘估计. 然而对于一个回归问题, 通常很难得到 大数量的重复. 例如 Jacquez 等 ${ }^{[5]}$ 强调在一个点得到超过 4 或 5 次重复通常是很不实际的, 2 或 3 次重复是常见的情形. 为此, $\mathrm{Chen}$ 和 $\mathrm{You}^{2)}$ 进一步讨论了当 $k \rightarrow \infty,\left\{n_{i}\right\}$ 为常数序列 时模型 (0.1) 的估计问题, 他们构造了 $\beta$ 的一个加权偏样条最小二乘估计.

因为存在大量的讨厌参数 $\operatorname{Var}\left(\varepsilon_{i}\right)=\sigma_{i}^{2}$, 所以当 $k \rightarrow \infty$ 时所有 $\sigma_{i}^{2}$ 的估计是不相合的, 并 且相应的加权偏样条最小二乘估计没有以已知方差 $\sigma_{i}^{2}$ 的倒数为权的加权偏样条最小二乘估计 有效, 因此似乎仍然有可能改进加权偏样条最小二乘估计. 本文提出了 $\beta$ 的一个迭代加权偏样 条最小二乘估计 (IWPSLSE), 此估计比加权偏样条最小二乘估计更为有效, 我们还建立了此估 计的渐近正态性. 另外，提出了一个自适应方法，该方法保证经过有限次迭代后，迭代过程会 终止，并且产生的估计渐近等价于使用迭代方法所能产生的最好的估计.

\section{1 迭代加权偏样条最小二乘估计方法}

本文假定设计点 $x_{i j}$ 是固定的 $\left(i=1, \cdots, k, j=1, \cdots, n_{i}\right)$. 另外，假定向量 $(1, \cdots, 1)^{\prime}$ 不 包含在由 $X=\left(x_{11}, \cdots, x_{1 n_{1}}, \cdots, x_{k n_{k}}\right)^{\prime}$ 的列向量张成的空间中，这样根据文献 [6] 保证了模型 $(0.1)$ 是可辨别的. 为了简单起见, 还假定 $t_{11} \leqslant \cdots \leqslant t_{1 n_{1}} \leqslant t_{21} \leqslant \cdots \leqslant t_{2 n_{2}} \leqslant \cdots \leqslant t_{k n_{k}}$ 和 $t_{i 1}-t_{i-1,1}=O\left(k^{-1}\right)$, 其中 $t_{01}=0, t_{k+1,1}=1$.

假定 $\left\{x_{i j}^{\prime}, t_{i j}, y_{i j} ; i=1, \cdots, k, j=1, \cdots, n_{i}\right\}$ 满足模型 $(0.1)$. 首先定义通过由 $B$ 样条级数 近似 $g(\cdot)$ 产生的 $\beta$ 的加权偏样条最小二乘估计. 为了描述估计方法, 先引入一些记号.

对于非负整数 $m$, 令 $0 \leqslant s_{1}^{*} \leqslant \cdots s_{m}^{*} \leqslant 1$ 和 $f(\cdot)$ 为 $[0,1]$ 上的一个函数, 再令 $\left[s_{1}^{*}, \cdots, s_{m}^{*}\right] f$ 为 $f(\cdot)$ 的差分, 该差分定义为

$$
\begin{gathered}
{\left[s_{1}^{*}\right] f=f\left(s_{1}^{*}\right), \quad\left[s_{1}^{*}, \cdots, s_{m}^{*}\right] f=\frac{\left[s_{2}^{*}, \cdots, s_{m}^{*}\right] f-\left[s_{1}^{*}, \cdots, s_{m-1}^{*}\right] f}{s_{m}^{*}-s_{1}^{*}}, \quad s_{m}^{*} \neq s_{1}^{*},} \\
{\left[s_{1}^{*}, \cdots, s_{m}^{*}\right] f=\frac{d^{m-1}}{d t^{m-1}} \frac{f\left(s_{1}^{*}\right)}{(m-1) !}, \quad s_{m}^{*}=s_{1}^{*} \in(0,1),} \\
{\left[s_{1}^{*}, \cdots, s_{m}^{*}\right] f=\frac{d_{+}^{m-1}}{d t^{m-1}} \frac{f\left(s_{1}^{*}\right)}{(m-1) !}, \quad s_{m}^{*}=s_{1}^{*}=0,} \\
{\left[s_{1}^{*}, \cdots, s_{m}^{*}\right] f=\frac{d_{-}^{m-1}}{d t^{m-1}} \frac{f\left(s_{1}^{*}\right)}{(m-1) !}, \quad s_{m}^{*}=s_{1}^{*}=1,}
\end{gathered}
$$

其中 $\frac{d_{-}^{m-1}}{d t^{m-1}}$ 和 $\frac{d_{+}^{m-1}}{d t^{m-1}}$ 分别表示第 $m-1$ 阶左、右导数算子.

对于非负整数 $\nu$ 和 $m_{k}=\left[k^{1 /(2 \nu+1)}\right]$ ([x] 表示 $x$ 的最大整数部分), 令 $\nu_{i}=i / m_{k}, i=$ $0,1, \cdots, m_{k}$ 和

$$
\begin{gathered}
0=s_{1}=\cdots=s_{\nu+1}, s_{\nu+2}=\nu_{1}, \cdots, s_{m_{k}+\nu}=\nu_{m_{k}-1}, s_{m_{k}+\nu+1}=\cdots=s_{m_{k}+2 \nu+1}=1, \\
B_{m_{k} j}(\cdot)=(-1)^{\nu+1}\left(s_{j+\nu+1}-s_{j}\right)\left[s_{j}, \cdots, s_{j+\nu+1}\right](\cdot-s)_{+}^{\nu}, \quad j=1, \cdots, m_{k}+\nu
\end{gathered}
$$

1) Liang H, Härdle W. Asymptotic properties of parametric estimation in partially linear heteroscedastic models. Technical Report No. 33, Sonderforschungsbereich 373, Humboldt-Universität zu Berlin, 1997

2) Chen G, You J. Delete-group jackknife estimate in partially linear regression models with heteroscedasticity. Technical Report, Department of Mathematics \& Statistics, University of Regina. 2002 
和

$$
B_{m_{k}}(\cdot)=\left(B_{m_{k} 1}(\cdot), \cdots, B_{m_{k}, m_{k}+\nu}(\cdot)\right)^{\prime}
$$

这里如果 $t<s$, 则定义 $(t-s)_{+}^{0}=0$; 否则 $(t-s)_{+}^{0}=1$ 且 $(t-s)_{+}^{\nu}=(t-s)^{\nu}(t-s)_{+}^{0}$. 由定义 可见 $B_{m_{k}}(\cdot)$ 是正则化 $B$ - 样条向量 (阶为 $\nu+1$ ), 相应的在 $[0,1]$ 上的扩展分割是 $s_{1}, \cdots, s_{m_{k}+\nu}$ (见文献 [7] p.224), 并且函数 $B_{m_{k} 1}(\cdot), \cdots, B_{m_{k}, m_{k}+\nu}(\cdot)$ 是在 $(0,1)$ 上 $\nu-1$ 次连续可微的.

根据文献 [7] 中的推论 $6.2 .1, g(\cdot)$ 可以由此基的一个线性组合 $\alpha^{\prime} B_{m_{k}}(\cdot)$ 来近似，其中 $\alpha \in$ $\mathbb{R}^{m_{k}+\nu}$. 对于 $\beta \in \mathbb{R}^{p}, \alpha \in \mathbb{R}^{m_{k}+\nu}$, 令

$$
S_{n}(\beta, \alpha)=n^{-1} \sum_{i=1}^{k} \sum_{j=1}^{n_{i}}\left(y_{i j}-x_{i j}^{\prime} \beta-\alpha^{\prime} B_{m_{k}}\left(t_{i j}\right)\right)^{2} .
$$

如果 $S_{n}(\cdot, \cdot)$ 在 $\hat{\beta}$ 和 $\hat{\alpha}$ 处极小化, 则有

$$
\hat{\beta}=\left(X^{\prime} M_{B_{m_{k}}} X\right)^{-1} X^{\prime} M_{B_{m_{k}}} y, \quad \hat{\alpha}=\left(B_{m_{k}}^{\prime} B_{m_{k}}\right)^{-1} B_{m_{k}}^{\prime}(y-X \hat{\beta}),
$$

其中 $y=\left(y_{11}, \cdots, y_{1 n_{1}}, \cdots, y_{k n_{k}}\right)^{\prime}, X=\left(x_{1}, \cdots, x_{p}\right), x_{s}=\left(x_{11 s}, \cdots, x_{1 n_{1} s}, \cdots, x_{k n_{k} s}\right)^{\prime}, s=$ $1, \cdots, p, M_{B_{m_{k}}}=I_{n}-B_{m_{k}}\left(B_{m_{k}}^{\prime} B_{m_{k}}\right)^{-1} B_{m_{k}}^{\prime}, B_{m_{k}}=\left(B_{m_{k}}\left(t_{11}\right), \cdots, B_{m_{k}}\left(t_{1 n_{1}}\right), \cdots, B_{m_{k}}\left(t_{k n_{k}}\right)\right)^{\prime}$ 和 $I_{n}$ 是 $n$ 阶单位矩阵. $\hat{\beta}$ 称为 $\beta$ 的偏样条最小二乘估计 (PSLSE).

根据 $\hat{\beta}, \hat{\alpha}$ 以及文献 [8] 中的二阶段估计方法，构造如下加权的偏样条最小二乘估计:

$\hat{\beta}_{w}=\left[X^{\prime} M_{B_{m_{k}}} \operatorname{diag}\left(w_{1} I_{n_{1}}, \cdots, w_{k} I_{n_{k}}\right) M_{B_{m_{k}}} X\right]^{-1} X^{\prime} M_{B_{m_{k}}} \operatorname{diag}\left(w_{1} I_{n_{1}}, \cdots, w_{k} I_{n_{k}}\right) M_{B_{m_{k}}} y$, 其中 $w_{i}^{-1}=n_{i}^{-1} \sum_{j=1}^{n_{i}}\left(y_{i j}-x_{i j}^{\prime} \hat{\beta}-B_{m_{k}}^{\prime}\left(t_{i j}\right) \hat{\alpha}\right)^{2}$.

对于固定的序列 $\left\{n_{i}\right\}, w_{i}^{-1}$ 不是 $\sigma_{i}^{2}$ 的相合估计, 因此仍然能够改进 $\hat{\beta}_{w}$. 为了改进 $\hat{\beta}_{w}$, 一 个自然的方法是下列迭代方法: 先得到 $\beta$ 的一个估计，再得到 $\sigma_{i}^{2}$ 的一个估计，然后重复此过 程. 此方法广泛地应用在线性回归中 (见文献 $[9,10]$ 以及其他文献).

现在把此方法应用到半参数回归模型 (0.1) 上，具体过程如下: 经过第 $s$ 次迭代后的迭代 加权偏样条最小二乘估计为

$$
\begin{aligned}
\hat{\beta}_{w}^{(s)}= & {\left[X^{\prime} M_{B_{m_{k}}} \operatorname{diag}\left(w_{1}^{(s)} I_{n_{1}}, \cdots, w_{k}^{(s)} I_{n_{k}}\right) M_{B_{m_{k}}} X\right]^{-1} } \\
& \times X^{\prime} M_{B_{m_{k}}} \operatorname{diag}\left(w_{1}^{(s)} I_{n_{1}}, \cdots, w_{k}^{(s)} I_{n_{k}}\right) M_{B_{m_{k}}} y
\end{aligned}
$$

其中 $w_{i}^{(s)}=\left[v_{i}\left(\hat{\beta}_{w}^{(s-1)}\right)\right]^{-1}, v_{i}\left(\hat{\beta}_{w}^{(s-1)}\right)=n_{i}^{-1} \sum_{i=1}^{n_{i}}\left(y_{i j}-x_{i j}^{\prime} \hat{\beta}_{w}^{(s-1)}-B_{m_{k}}^{\prime}\left(t_{i j}\right) \hat{\alpha}_{w}^{(s-1)}\right)^{2}$ 和

$$
\hat{\alpha}_{w}^{(s-1)}=B_{m_{k}}^{\prime}\left(t_{i j}\right)\left(B_{m_{k}}^{\prime} B_{m_{k}}\right)^{-1} B_{m_{k}}^{\prime}\left(y-X \hat{\beta}_{w}^{(s-1)}\right) \text {. }
$$

在一些实际问题中, 我们经常感兴趣的不仅是 $\beta$ 本身, 而且是 $\beta$ 的某个函数. 例如, 如果 模型 (0.1) 中的第 1 项是多项式, 我们可能更关心的是此多项式的根和拐点. 如 $\beta$ 的函数 $m(\beta)$, 一个自然的估计是 $m\left(\hat{\beta}_{w}^{(s)}\right)$, 这里 $m(\cdot)$ 为 $\mathbb{R}^{p}$ 到 $\mathbb{R}^{q}$ 上的函数, 并且在点 $\beta$ 可微, 下面两节将研 究 $m\left(\hat{\beta}_{w}^{(s)}\right)$ 的渐近性质.

\section{2 渐近正态性}

为了导出迭代加权偏样条最小二乘估计 $m\left(\hat{\beta}_{w}^{(s)}\right)$ 的渐近正态性, 先列出一些记号和假定.

$$
\varepsilon_{i}=\left(\varepsilon_{i 1}, \varepsilon_{i 2}, \cdots, \varepsilon_{i n_{i}}\right)^{\prime}, \quad \varepsilon=\left(\varepsilon_{1}^{\prime}, \varepsilon_{2}^{\prime}, \cdots, \varepsilon_{k}^{\prime}\right)^{\prime}, \quad e_{i}=\varepsilon_{i}^{\prime} \varepsilon_{i} / n_{i}
$$




$$
\tilde{\varepsilon}_{i}=\varepsilon_{i} / e_{i}, \quad \tilde{\varepsilon}=\left(\tilde{\varepsilon}_{1}^{\prime}, \tilde{\varepsilon}_{2}^{\prime}, \cdots, \tilde{\varepsilon}_{k}^{\prime}\right)^{\prime}, \quad V_{i}=\operatorname{Cov}\left(\varepsilon_{i}\right), \quad V=\operatorname{diag}\left(V_{1}, V_{2}, \cdots, V_{k}\right)
$$

下列假定将在全文中用到，这些假定尽管看上去非常冗长，实际上它们非常弱，并且易于 满足 (见注记 $2.1 \sim 2.3$ ).

假定 2.1 存在正常数 $\sigma_{0}, \sigma_{\infty}$ 和正整数 $n_{0}, n_{\infty}$, 使得对于所有的 $i$, 有

$$
\sigma_{0} I_{n_{i}} \leqslant V_{i} \leqslant \sigma_{\infty} I_{n_{i}}, \quad n_{0} \leqslant n_{i} \leqslant n_{\infty} .
$$

假定 2.2 存在正常数 $c$ 和 $1 /(2 \nu)<\delta<1 / 2$, 使得对于所有的 $i=1, \cdots, k, j=1, \cdots, n_{i}$ ， 有

$$
E\left(\frac{\varepsilon_{i j}}{\sum_{j=1}^{n_{i}} \varepsilon_{i j}^{2}}\right)=0, \quad E\left|\varepsilon_{i j}\right|^{2+\delta} \leqslant c, \quad E\left(\sum_{j=1}^{n_{i}} \varepsilon_{i j}^{2}\right)^{-(1+\delta)} \leqslant c
$$

其中 $\nu$ 将在假定 2.4 中定义.

假定 2.3 存在 $[0,1]$ 上的有界函数 $h_{s}(\cdot)(s=1, \cdots, p)$, 使得

$$
x_{i j s}=h_{s}\left(t_{i j}\right)+u_{i j s}, i=1, \cdots, k, j=1, \cdots, n_{i}, \quad s=1, \cdots, p,
$$

其中 $u_{i j s}$ 满足

$$
\lim _{k \rightarrow \infty} \frac{\sum_{i=1}^{k} \sum_{j=1}^{n_{i}} u_{i j s_{1}} u_{i j s_{2}}}{n}=b_{s_{1} s_{2}}, \quad s_{1}=1, \cdots, p, \quad s_{2}=1, \cdots, p,
$$

并且矩阵 $B=\left(b_{s_{1} s_{2}}\right)$ 是非退化的; 另外令 $v_{1}=u_{11}, \cdots, v_{n_{1}}=u_{1 n_{1}}, \cdots, v_{n}=u_{k n_{k}}$, 则对于 $(1, \cdots, n)$ 的任意排列 $\left(j_{1}, \cdots, j_{n}\right)$, 有

$$
\limsup _{k \rightarrow \infty} \frac{1}{\sqrt{n} \log n} \max _{1 \leqslant m \leqslant n}\left\|\sum_{i=1}^{m} v_{j_{i}}\right\|<\infty
$$

其中 $\|\cdot\|$ 表示 Euclid 模.

假定 2.4 假定函数 $g(\cdot)$ 和 $h_{s}(\cdot)(s=1, \cdots, p)$ 在 $[0,1]$ 上 $\nu$ 次连续可微，其中 $\nu>1$.

注记 2.1 (2.1) 式中第 1 项成立的充分条件是 $\varepsilon_{i j}$ 的分布关于 0 对称. 如果 $\varepsilon_{i j}$ 是正态分 布 $N\left(0, \sigma_{i}^{2}\right)$ 并且 $n_{i} \geqslant n_{0}=3$, 则 $(2.1)$ 式中的第 2 、第 3 项成立. 如果 $\varepsilon_{i j}$ 有密度

$$
f_{i j}(z)=[\Gamma(\alpha)]^{-1} \lambda_{i}^{-\alpha}|z|^{2 \alpha-1} \exp \left(-z^{2} / \lambda_{i}\right),
$$

其中 $\alpha>0$ 和 $\lambda_{i}=\sigma_{i}^{2} / \alpha$, 则 (2.1) 式中第 2 、第 3 项成立的充要条件是 $n_{i} \alpha \geqslant n_{0} \alpha>1$. 另外, 如果 $n_{i} \geqslant n_{0}=3$ 并且 $\varepsilon_{i j}$ 有密度 $f_{i}(z)$ 关于 0 对称, 并且满足 $\int|z|^{2+\delta} f_{i}(z) d z \leqslant c$ 和 $f_{i}(z) \leqslant c$, 这里 $z \in[-a, a], a$ 和 $c$ 为与 $i$ 无关的正常数，则 (2.1) 式中的第 2 、第 3 项成立，具体细节可 见文献 [11].

注记 2.2 上述 $u_{i j s}$ 很像均值为 0 、不相关的随机变量, 并且 $h_{s}\left(t_{i j}\right)$ 为 $x_{i j s}$ 关于 $t_{i j}$ 的回 归函数. 特别地, 如果假定设计点 $\left(x_{i j}^{\prime}, t_{i j}\right)$ 为独立同分布随机变量, 并且令 $h_{s}\left(t_{i j}\right)=E\left(x_{i j s} \mid t_{i j}\right)$ 和 $u_{i j s}=x_{i j s}-h_{s}\left(t_{i j}\right)$, 另外 $E u_{i j} u_{i j}^{\prime}=B$, 则根据大数定律和重对数律, (2.3) 和 (2.4) 式以概 率 1 成立.

注记 2.3 假定 2.4 非常弱，通常的多项式函数和三角函数都满足此假定.

有了以上假定，可以建立上节提出的估计的渐近正态性. 定理 2.1 给出了迭代加权偏样条 最小二乘估计 $\hat{\theta}_{w}^{(s)}=m\left(\hat{\beta}_{w}^{(s)}\right)$ 在一般的组间协方差矩阵 $V_{i}(i=1, \cdots, k)$ 下的渐近正态性. 为简 单起见, 假定 $q=1$, 我们的结果很容易推广到 $q>1$ 的情形. 
定理 2.1 如果假定 $2.1 \sim 2.4$ 成立, $\hat{\beta}_{w}^{(0)}=\hat{\beta}$ 并且 $m(\cdot)$ 在 $\beta$ 处可微, 则对于任意固定的 $s$, 当 $n \rightarrow \infty$ 时有

$$
\left\{[\nabla m(\beta)]^{\prime} G_{k}^{-1} \Sigma_{k}^{(s)} G_{k}^{-1} \nabla m(\beta)\right\}^{-\frac{1}{2}}\left(\hat{\theta}_{w}^{(s)}-\theta\right) \rightarrow_{D} N(0,1),
$$

其中 “ $\rightarrow D$ ” 表示以分布收敛, $\Sigma_{k}^{(s)}=\operatorname{Cov}\left(A_{k}^{(s)} \tilde{\varepsilon}+C_{k}^{(s)} \varepsilon\right), \nabla m(\cdot)$ 是 $m(\cdot)$ 的方向导数, $A_{k}^{(s)}=$ $\sum_{l=0}^{s-1}\left(2 H_{k} G_{k}^{-1}\right)^{l} U^{\prime}, C_{k}^{(s)}=\left(2 H_{k} G_{k}^{-1}\right)^{s} C_{k}^{(0)}, C_{k}^{(0)}=G_{k}\left(U^{\prime} U\right)^{-1} U^{\prime}, G_{k}=\sum_{i=1}^{k} E\left(e_{i}^{-1}\right) U_{i}^{\prime} U_{i}, H_{k}=$ $E \sum_{i=1}^{k} n_{i}^{-1} U_{i}^{\prime} \tilde{\varepsilon}_{i} \tilde{\varepsilon}_{i}^{\prime} U_{i}, U_{i}=\left(u_{i 1}, \cdots, u_{i n_{i}}\right)^{\prime}$ 和 $U=\left(U_{1}^{\prime}, \cdots, U_{k}^{\prime}\right)$.

定理 2.1 的证明将在附录中给出.

如果 $\varepsilon_{i j} / \sigma_{i}\left(j=1, \cdots, n_{i} ; i=1, \cdots, k\right)$ 为独立同分布的随机变量, $n_{i}=n_{0}$ 并且当 $j \neq j^{\prime}$ 时 $E\left(\varepsilon_{i j} \varepsilon_{i j^{\prime}}\right) / e_{i}^{2}=0$, 则 $H_{k}=n_{0}^{-1} G_{k}, G_{k}=\rho\left(n_{0}\right) U^{\prime} V^{-1} U$, 其中 $\rho\left(n_{0}\right)=\sigma_{i}^{2} E e_{i}^{-1}$,

$$
A_{k}^{(s)}=\left(1-\frac{2}{n_{0}}\right)^{-1}\left[1-\left(\frac{2}{n_{0}}\right)^{s}\right] U^{\prime}+\left(\frac{2}{n_{0}}\right)^{s} A_{k}^{(0)}, \quad C_{k}^{(s)}=\left(\frac{2}{n_{0}}\right)^{s} C_{k}^{(0)} .
$$

进一步, 如果当 $j \neq j^{\prime}$ 时有 $E\left(\varepsilon_{i j} \varepsilon_{i j^{\prime}}\right) / e_{i}=0$, 则

$$
\Sigma_{k}^{(s)}=A_{k}^{(s)} E\left(\tilde{\varepsilon} \tilde{\varepsilon}^{\prime}\right)\left(A_{k}^{(s)}\right)^{\prime}+A_{k}^{(s)}\left(C_{k}^{(s)}\right)^{\prime}+C_{k}^{(s)}\left(A_{k}^{(s)}\right)^{\prime}+C_{k}^{(s)} \operatorname{diag}\left(\sigma_{1}^{2} I_{n_{1}}, \cdots, \sigma_{k}^{2} I_{n_{k}}\right)\left(C_{k}^{(s)}\right)^{\prime} .
$$

定理 2.2 如果假定 $2.1 \sim 2.4$ 成立, 并且当 $j \neq j^{\prime}$ 时 $E\left(\varepsilon_{i j} \varepsilon_{i j^{\prime}}\right) / e_{i}^{2}=0, E\left(\varepsilon_{i j} \varepsilon_{i j^{\prime}}\right) / e_{i}=0$. 另外还假定 $\varepsilon_{i j} / \sigma_{i}\left(j=1, \cdots, n_{i} ; i=1, \cdots, k\right)$ 为独立同分布随机变量, $n_{i}=n_{0}, \hat{\beta}_{w}^{(0)}=\hat{\beta}$, 并且 $m(\cdot)$ 在 $\beta$ 可微, 则对于任意的 $s$, 有

$$
\left(\tau_{k}^{(s)} / k\right)^{-\frac{1}{2}}\left(\hat{\theta}_{w}^{(s)}-\theta\right) \rightarrow_{D} N(0,1)
$$

其中

$$
\begin{aligned}
\tau_{k}^{(s)}= & k\left\{\varphi(s) / \rho\left(n_{0}\right)(\nabla m(\beta))^{\prime}\left[U^{\prime} \operatorname{diag}\left(\sigma_{1}^{-2} I_{n_{1}}, \cdots, \sigma_{k}^{-2} I_{n_{k}}\right) U\right]^{-1} \nabla m(\beta)\right. \\
& \left.+\psi(s)(\nabla m(\beta))^{\prime}\left(U^{\prime} U\right)^{-1} U^{\prime} \operatorname{diag}\left(\sigma_{1}^{2} I_{n_{1}}, \cdots, \sigma_{k}^{2} I_{n_{k}}\right) U\left(U^{\prime} U\right)^{-1} \nabla m(\beta)\right\},
\end{aligned}
$$

这里

$$
\varphi(s)=\left(1-\frac{2}{n_{0}}\right)^{-2}\left[1-\left(\frac{2}{n_{0}}\right)^{s}\right]^{2}+2\left(1-\frac{2}{n_{0}}\right)^{-1}\left[1-\left(\frac{2}{n_{0}}\right)^{s}\right]\left(\frac{2}{n_{0}}\right)^{s}, \psi(s)=\left(\frac{2}{n_{0}}\right)^{2 s} .
$$

为应用定理 2.1 和 2.2 作统计推断, 需要 $[\nabla m(\beta)]^{\prime} G_{k}^{-1} \Sigma_{k}^{(s)} G_{k}^{-1} \nabla m(\beta)$ 和 $\tau_{k}^{(s)}$ 的相合估 计. 如文献 [12] 所指出的一样, 传统的 $\delta$ 法和刀切法无法提供 $[\nabla m(\beta)]^{\prime} G_{k}^{-1} \Sigma_{k}^{(s)} G_{k}^{-1} \nabla m(\beta)$ 的 相合估计，类似于 Chen 和 You 文 ${ }^{1)}$ 可以用删群刀切法构造 $[\nabla m(\beta)]^{\prime} G_{k}^{-1} \Sigma_{k}^{(s)} G_{k}^{-1} \nabla m(\beta)$ 的 相合估计. 对于 $\tau_{k}^{(s)}$, 可以用 $\delta$ 法或者刀切法构造它的相合估计 (通过删除偏残差, 见引理 3.1).

\section{3 渐近有效性和自适应的估计方法}

现在研究 $\hat{\theta}_{w}^{(s)}$ 的渐近有效性, 为简单起见, 我们的注意力放在 $n_{i}=n_{0}$ 和 $\varepsilon_{i j} / \sigma_{i}$ 为独立 同分布的情形. 令

$$
c_{k}=k\left[(\nabla m(\beta))^{\prime}\left(U^{\prime} U\right)^{-1}\left(U^{\prime} V U\right)\left(U^{\prime} U\right)^{-1} \nabla m(\beta)\right], \quad \tilde{c}_{k}=k\left[(\nabla m(\beta))^{\prime}\left(U^{\prime} V^{-1} U\right)^{-1} \nabla m(\beta)\right] .
$$

则根据定理 2.2 , 对于固定的 $s, \hat{\theta}_{w}^{(s)}$ 的渐近方差为 $\tau_{k}^{(s)} / k=\left(\varphi(s) / \rho\left(n_{0}\right) \tilde{c}_{k}+\psi(s) c_{k}\right) / k$. 
定理 3.1 假定 $c=\lim _{k \rightarrow \infty} c_{k}$ 和 $\tilde{c}=\lim _{k \rightarrow \infty} \tilde{c}_{k}$ 存在, 则在定理 2.2 的条件下存在整数 $s^{*}$, 使得对于所有 $s, \lim _{k \rightarrow \infty} \tau_{k}^{\left(s^{*}\right)} / \tau_{k}^{(s)} \leqslant 1$ 且等号至多在 $s=s^{*}$ 或 $s^{*}+1$ 时成立.

定理 3.1 的证明类似于文献 [9] 中定理 4 的证明，此处从略.

注记 3.1 由定理 3.1 可见, $\hat{\theta}_{w}^{\left(s^{*}\right)}$ 是 $\theta$ 在 $\hat{\theta}_{w}^{(s)}(s=1,2, \cdots)$ 中的最优估计, 并且在渐近 方差意义下 $s^{*}$ 是最优迭代数.

最优的 $s^{*}$ 通常是未知的, 因此需要用数据去估计它. 令 $a_{k}=\tilde{c}_{k} / \rho\left(n_{0}\right)$,

$$
\hat{a}_{k}=k \sum_{i=1}^{k} \sum_{j=1}^{n_{i}}\left(1-h_{i j}\right)\left(\hat{\theta}_{w}^{(i, j)}-\hat{\theta}_{w}^{(1)}\right)^{2}, \quad \hat{c}_{k}=k \sum_{i=1}^{k} \sum_{j=1}^{n_{i}}\left(1-c_{i j}\right)\left(\hat{\theta}_{0}^{(i, j)}-\hat{\theta}_{0}\right)^{2},
$$

其中

$$
\begin{gathered}
h_{i j}=n_{i}^{-1} v_{i}^{-1} \hat{x}_{i j}^{\prime}\left[X^{\prime} M_{B_{m_{k}}} \operatorname{diag}\left(v_{1}^{-1} I_{n_{1}}, \cdots, v_{k}^{-1} I_{n_{k}}\right) M_{B_{m_{k}}} X\right]^{-1} \hat{x}_{i j}, \quad v_{i}=v_{i}(\hat{\beta}), \\
M_{B_{m_{k}}} X=\left(\hat{x}_{i j}\right)_{n \times p}, \quad c_{i j}=n_{i}^{-1} \hat{x}_{i j}^{\prime}\left(X^{\prime} M_{B_{m_{k}}} X\right)^{-1} \hat{x}_{i j}, \quad \hat{\theta}_{w}^{(i, j)}=m\left(\hat{\beta}_{w}^{(i, j)}\right), \\
\hat{\beta}_{w}^{(i, j)}=\hat{\beta}_{w}-\left(1-h_{i j}\right)^{-1}\left[X^{\prime} M_{B_{m_{k}}} \operatorname{diag}\left(v_{1}^{-1} I_{n_{1}}, \cdots, v_{k}^{-1} I_{n_{k}}\right) M_{B_{m_{k}}} X\right]^{-1} \hat{x}_{i j}\left(\hat{y}_{i j}-\hat{x}_{i j}^{\prime} \hat{\beta}_{w}\right)
\end{gathered}
$$

和

$$
\hat{\theta}_{0}^{(i, j)}=m\left(\hat{\beta}^{(i, j)}\right), \quad \hat{\beta}^{(i, j)}=\hat{\beta}-\left(1-c_{i j}\right)^{-1}\left(X^{\prime} M_{B_{m_{k}}} X\right)^{-1} \hat{x}_{i j}\left(\hat{y}_{i j}-\hat{x}_{i j}^{\prime} \hat{\beta}\right) .
$$

引理 3.1 在定理 3.1 的条件下有 $\hat{a}_{k}-a_{k} \rightarrow_{p} 0$ 和 $\hat{c}_{k}-c_{k} \rightarrow_{p} 0$.

证 仅证明 $\hat{c}_{k}-c_{k} \rightarrow_{p} 0$, 证明 $\hat{a}_{k}-a_{k} \rightarrow_{p} 0$ 是类似的. 令 $\widehat{U}=\sum_{i=1}^{k} \sum_{j=1}^{n_{i}}\left(1-c_{i}\right)\left(\hat{\beta}^{(i, j)}-\right.$ $\hat{\beta})\left(\hat{\beta}^{(i, j)}-\hat{\beta}\right)^{\prime}$. 类似于 You 文 ${ }^{1)}$ 中定理 3.1.6 的证明, 有

$$
k \widehat{U}-k\left(U^{\prime} U\right)^{-1} U^{\prime} V U\left(U^{\prime} U\right)^{-1} \rightarrow_{p} 0 .
$$

令 $G\left(\xi_{(i, j)}\right)$ 为 $p$ 维行向量, 其第 $l$ 个元素是 $m$ 在 $\xi_{(i, j)}^{(l)}$ 的方向导数, 这里 $\xi_{(i, j)}^{(l)}$ 位于 $\hat{\beta}^{(i, j)}$ 和 $\hat{\beta}$ 之 间. 又令 $D_{(i, j)}=\nabla m(\hat{\beta})\left(\hat{\beta}^{(i, j)}-\hat{\beta}\right), Q_{(i, j)}=\left[G\left(\xi_{(i, j)}\right)-\nabla m(\hat{\beta})\right]\left(\hat{\beta}^{(i, j)}-\hat{\beta}\right), D=\left(\left(1-c_{i}\right) k D_{(i, j)}\right)$ 和 $Q=\left(\left(1-c_{i}\right) k Q_{(i, j)}\right)$, 则

$$
\hat{c}_{k}=D^{\prime} D+Q^{\prime} Q+D^{\prime} Q+Q^{\prime} D .
$$

由 $D^{\prime} D=k(\nabla m(\hat{\beta}))^{\prime} \widehat{U} \nabla m(\hat{\beta})$ 和 (3.1) 式及 $\nabla m(\cdot)$ 在 $\beta$ 这一点的连续性, 有

$$
D^{\prime} D-k(\nabla m(\beta))^{\prime}\left(U^{\prime} U\right)^{-1} U^{\prime} V U\left(U^{\prime} U\right)^{-1} \nabla m(\beta)=o_{p}(1) .
$$

由 Cauchy-Schwarz 不等式, 仅需证 $Q^{\prime} Q=o_{p}(1)$. 对于任意的 $\delta_{1}>0$, 存在 $\delta_{2}>0$, 使得当 $\|x-\beta\|+\|y-\beta\| \leqslant \delta_{2}$ 时, $\|\nabla m(x)-\nabla m(y)\| \leqslant \delta_{1}$. 记

$$
A_{n}^{\delta_{2}}=\left\{\|\hat{\beta}-\beta\| \leqslant \delta_{2} / 3,\left\|\hat{\beta}^{(i, j)}-\beta\right\|<\delta_{2} / 3 \text { 对于所有 } i=1, \cdots, k, j=1, \cdots, n_{i}\right\} \text {, }
$$

则

$$
\operatorname{tr}\left(I_{A_{n}^{\delta_{2}}} Q^{\prime} Q\right)=k \sum_{i=1}^{k} \sum_{i=1}^{n_{i}}\left(1-c_{i}\right)^{-1}\left\|\left[G\left(\xi_{(i, j)}\right)-\nabla m(\hat{\beta})\right]\left(\hat{\beta}^{(i, j)}-\hat{\beta}\right)\right\|^{2} I_{A_{n}^{\delta_{2}}} \leqslant \delta_{1}^{2} n \operatorname{tr}(\widehat{U}),
$$

其中 $I_{A}$ 是 $A$ 的示性函数, 因此对于任意给定的 $\delta_{0}$, 有

$$
P\left(\operatorname{tr}\left(Q^{\prime} Q\right)>\delta_{0}\right) \leqslant 1-P\left(A_{n}^{\delta_{2}}\right)+P\left(n \operatorname{tr}(\widehat{U})>\delta_{0} / \delta_{1}^{2}\right) .
$$

1) You J. Semiparametric regression models with serially correlated and/or heteroscedastic errors. Unpublished Ph D thesis, University of Regina. 2002 
类似于 You 文 ${ }^{1)}$ 中引理 3.1 .11 的证明, $\max _{1 \leqslant i \leqslant k, 1 \leqslant j \leqslant n_{i}}\left\|\hat{\beta}^{(i, j)}-\hat{\beta}\right\| \rightarrow_{p} 0$ 成立. 这意味着对 于任意 $\delta>0$,

$$
\lim _{n \rightarrow \infty} P\left(\|\hat{\beta}-\beta\|<\delta,\left\|\hat{\beta}^{(i, j)}-\hat{\beta}\right\| \leqslant \delta \text { 对于所有 } i=1, \cdots, k, j=1, \cdots, n_{i}\right)=1,
$$

因此

$$
0 \leqslant \limsup _{n \rightarrow \infty} P\left(\operatorname{tr}\left(Q^{\prime} Q\right)>\delta_{0}\right) \leqslant \limsup _{n \rightarrow \infty} P\left(n \operatorname{tr}(\widehat{U})>\delta_{0} / \delta_{1}^{2}\right),
$$

由 (3.1) 式和选择小的 $\delta_{1}$, 上式右边可以足够小, 即 $Q^{\prime} Q=o_{p}(1)$. 证毕.

令 $\nabla(s)=[\varphi(s+1)-\varphi(s)] /[\psi(s)-\psi(s+1)], \hat{\gamma}_{k}=\hat{c}_{k} / \hat{a}_{k}$ 和 $\gamma_{k}=c_{k} / a_{k}$, 则由引理 3.1, $\hat{\gamma}_{k}-\gamma_{k} \rightarrow_{p} 0$ 成立. 定义 $\hat{s}_{k}=\min \left\{s: \nabla(s) \geqslant \hat{\gamma}_{k}, s=0,1,2, \cdots\right\}$. 下面定理表明 $\hat{s}_{k}$ 是 $s^{*}$ 的一个 相合估计.

定理 3.2 在定理 3.1 的条件下有 $\lim _{k \rightarrow \infty} P\left(\hat{s}_{k}=s^{*}\right.$ 或 $\left.\hat{s}_{k}=s^{*}+1\right)=1$. 进一步地, 如 果 $s^{*}$ 是惟一的最优解 $\left(\lim _{k \rightarrow \infty} \tau_{k}^{\left(s^{*}+1\right)} / \tau_{k}^{\left(s^{*}\right)}>1\right)$, 则 $\lim _{k \rightarrow \infty} P\left(\hat{s}_{k}=s^{*}\right)=1$.

证明类似于文献 [9] 定理 5 的证明.

定理 3.2 表明当 $k$ 足够大时，经过 $\hat{s}_{k}$ 次迭代后，如果迭代停止，则停止总是合适的. 这 导致了 $\theta$ 的如下自适应估计: $\hat{\theta}_{a}=\hat{\theta}_{w}^{\left(\hat{s}_{k}\right)}$.

根据定理 3.2 立即有

定理 3.3 若定理 3.2 中的条件成立, 则

(i) 当 $k \rightarrow \infty$ 时, $P\left(\hat{\theta}_{a}=\hat{\theta}_{w}^{\left(s^{*}\right)}\right.$ 或 $\left.\hat{\theta}_{a}=\hat{\theta}_{w}^{\left(s^{*}+1\right)}\right) \rightarrow 1$. 另外, 如果 $s^{*}$ 是惟一的最优解, 则 $P\left(\hat{\theta}_{a}=\hat{\theta}_{w}^{\left(s^{*}\right)}\right) \rightarrow 1$.

(ii) 令 $\tau^{*}=\lim _{k \rightarrow \infty} \tau_{k}^{\left(s^{*}\right)}$, 则 $\hat{\tau}_{k}-\tau^{*} \rightarrow_{p} 0$, 其中 $\hat{\tau}_{k}=\varphi\left(\hat{s}_{k}\right) \hat{a}_{k}+\psi\left(\hat{s}_{k}\right) \hat{c}_{k}$.

根据定理 $3.1 \sim 3.3$, 可知此自适应方法保证经过有限多次迭代后迭代过程终止, 并且产生 的估计渐近等价于通过迭代方法所能产生的最好的估计.

\section{4 结束语}

本文提出了参数分量 $\beta$ 的一个迭代加权偏样条最小二乘估计, 该估计比加权偏样条最小 二乘估计更加渐近有效. 我们还建立了此迭代加权偏样条最小二乘估计的渐近正态性, 这样就 能应用该估计做出更好的统计推断.

\section{参考文献}

1 Donnelly C A, Laird N M, Ware J H. Prediction and creation of smooth curves for temporally correlated longitudinal data. J Amer Statist Assoc, 1995, 90: 984 989

2 Zeger S L, Diggle P J. Semiparametric models for longitudinal data with application to CD4 cell numbers in HIV seroconverters. Biometrics, 1994, 50: 689 699

3 Zhang D, Lin X, Raz J, et al. Semiparametric stochastic mixed models for longitudinal data. J Amer Statist Assoc, 1998, 93: 710 719

4 Härdle W, Liang H, Gao J T. Partially Linear Models. Heidelberg: Physica-Verlag, 2000

5 Jacquez J A, Mather F J, Grawford C R. Linear regression with nonconstant, unknown error variances: simpling experiments with least squares weighted least squares and maximum likelihood estimators. Biometrics, 1968, 24: 607 627 
6 Chen H. Convergence rates for parametric components in a partly linear model. Ann Statist, 1988, 16: $136 \sim 146$

7 Schumaker L L. Spline Functions. New York: Wiley, 1981

8 Fuller W A, Rao J N K. Estimation for a linear regression model with unknown diagonal covariance matrix. Ann Statist, 1978, 6: 1149 1158

9 Chen J H, Shao J. Iterative weighted least squares estimators. Ann Statist, 1993, 21: 1071 1092

10 Hooper P M. Iterative weighted least squares estimation in heteroscedastic linear models. J Amer Statist Assoc, 1993, 88: 179 184

11 Shao J. Asymptotic distribution of the weighted least squares estimator. Ann Inst Statist Math, 1989, 41: $365 \sim 382$

12 Shao J, Rao J N K. Jackknife inference for heteroscedastic linear regression models. The Canadian Journal of Statistics, 1993, 21: 377 395

13 Burman P. Regression function estimation from dependent observations. J Multivariate Anal, 1991, 36: $263 \sim 279$

\section{附录}

在此附录中将证明第 2 节中的定理 2.1. 为此先介绍几个引理.

引理 1 如果函数 $f(\cdot)$ 满足假定 2.4 , 则有

$$
\sup _{t \in[0,1]}\left|f(t)-B_{m_{k}}(t)\left(B_{m_{k}}^{\prime} B_{m_{k}}\right)^{-1} B_{m_{k}}^{\prime} f\right|=O\left(n^{-1} m_{k}\right)+O\left(m_{k}^{-\nu}\right)
$$

其中 $f=\left(f\left(t_{11}\right), \cdots, f\left(t_{1 k_{1}}\right), \cdots, f\left(t_{k n_{k}}\right)\right)^{\prime}$.

引理 2 对于 $B$ 样条的基函数, 有

i) 对于所有的 $t, \sum_{i=1}^{m_{k}+\nu} B_{m_{k} i}^{2}(t) \leqslant 1$;

ii) $n^{-1} B_{m_{k}}^{\prime} B_{m_{k}}$ 的所有特征根介于 $c_{1} m_{k}^{-1}$ 和 $c_{2} m_{k}^{-1}$ 之间, 其中 $0<c_{1}<c_{2}<\infty$.

引理 1 和 2 的证明见文献 [13].

引理 3 若假定 $2.1 \sim 2.4$ 成立, $\hat{\beta}_{w}^{(1)}$ 为经过 1 次迭代后的迭代加权偏样条最小二乘估计 $\left(w_{i}=\left[v_{i}\left(\hat{\beta}_{w}^{(0)}\right)\right]^{-1}\right)$, 即

$$
\hat{\beta}_{w}^{(1)}=\left[X^{\prime} M_{B_{m_{k}}} \operatorname{diag}\left(w_{1} I_{n_{1}}, \cdots, w_{k} I_{n_{k}}\right) M_{B_{m_{k}}} X\right]^{-1} X^{\prime} M_{B_{m_{k}}} \operatorname{diag}\left(w_{1} I_{n_{1}}, \cdots, w_{k} I_{n_{k}}\right) M_{B_{m_{k}}} y,
$$

其中 $\hat{\beta}_{w}^{(0)}$ 满足 $\hat{\beta}_{w}^{(0)}-\beta=G_{k}^{-1}\left(A_{k} \tilde{\varepsilon}+C_{k} \varepsilon\right)+o_{p}\left(k^{-\frac{1}{2}}\right), G_{k}=\sum_{i=1}^{k} E\left(e_{i}^{-1}\right) U_{i}^{\prime} U_{i}, A_{k}$ 和 $C_{k}$ 满足 $A_{k} A_{k}^{\prime}=O(k)$, $C_{k} C_{k}^{\prime}=O(k)$, 则

$$
\hat{\beta}_{w}^{(1)}-\beta=G_{k}^{-1}\left(\tilde{A}_{k} \tilde{\varepsilon}+\tilde{C}_{k} \varepsilon\right)+o_{p}\left(k^{-\frac{1}{2}}\right),
$$

此处 $\tilde{A}_{k}=U^{\prime}+2 H_{k} G_{k}^{-1} A_{k}, \tilde{C}_{k}=2 H_{k} G_{k}^{-1} C_{k}, H_{k}=E\left(\sum_{i=1}^{k} n_{i}^{-1} U_{i}^{\prime} \tilde{\varepsilon}_{i} \tilde{\varepsilon}_{i}^{\prime} U_{i}\right)$ 和 $U=\left(U_{1}^{\prime}, \cdots, U_{k}^{\prime}\right)$.

证 令 $v_{i}=v_{i}\left(\hat{\beta}_{w}^{(0)}\right)$, 则根据 $\hat{\beta}_{w}^{(1)}$ 的定义有

$$
\begin{aligned}
\hat{\beta}_{w}^{(1)}-\beta= & {\left[X^{\prime} M_{B_{m_{k}}} \operatorname{diag}\left(v_{1}^{-1} I_{n_{1}}, \cdots, v_{k}^{-1} I_{n_{k}}\right) M_{B_{m_{k}}} X\right]^{-1} X^{\prime} M_{B_{m_{k}}} } \\
& \cdot \operatorname{diag}\left(v_{1}^{-1} I_{n_{1}}, \cdots, v_{k}^{-1} I_{n_{k}}\right)\left(\varepsilon+P_{B_{m_{k}}} \varepsilon+M_{B_{m_{k}}} g\right) \hat{=} I_{1}+I_{2}+I_{3},
\end{aligned}
$$

其中 $\varepsilon=\left(\varepsilon_{11}, \cdots, \varepsilon_{1 k_{1}}, \cdots, \varepsilon_{k n_{k}}\right)^{\prime}, g=\left(g\left(t_{11}\right), \cdots, g\left(t_{1 k_{1}}\right), \cdots, g\left(t_{k n_{k}}\right)\right)^{\prime}$. 我们将证明

$$
I_{1}=G_{k}^{-1}\left(\tilde{A}_{k} \tilde{\varepsilon}+\tilde{B}_{k} \varepsilon\right)+o_{p}\left(k^{-\frac{1}{2}}\right), \quad I_{i}=o_{p}\left(k^{-\frac{1}{2}}\right), i=2,3 .
$$

根据 $v_{i}$ 的定义有

$$
v_{i}^{-1}=e_{i}^{-1}+2 n_{i}^{-1} e_{i}^{-2} \sum_{j=1}^{n_{i}} \varphi_{i j} \varepsilon_{i j}-n_{i}^{-1} e_{i}^{-2} \sum_{j=1}^{n_{i}} \varphi_{i j}^{2}+e_{i}^{-2} v_{i}^{-1}\left(v_{i}-e_{i}\right)^{2},
$$

其中 $\varphi_{i j}=x_{i j}^{\prime}\left(\hat{\beta}_{w}^{(0)}-\beta\right)+\left(B_{m_{k}}^{\prime}\left(t_{i j}\right) \hat{\alpha}_{w}^{(0)}-g\left(t_{i j}\right)\right), \hat{\alpha}_{w}^{(0)}=\left(B_{m_{k}}^{\prime} B_{m_{k}}\right)^{-1} B_{m_{k}}^{\prime}\left(y-X \hat{\beta}_{w}^{(0)}\right)$. 先证明

$$
X^{\prime} M_{B_{m_{k}}} \operatorname{diag}\left[e_{1}^{-2} v_{1}^{-1}\left(v_{1}-e_{1}\right)^{2} I_{n_{1}}, \cdots, e_{k}^{-2} v_{k}^{-1}\left(v_{k}-e_{k}\right)^{2} I_{n_{k}}\right] \varepsilon=o_{p}\left(k^{\frac{1}{2}}\right)
$$


和

$$
X^{\prime} M_{B_{m_{k}}} \Phi \operatorname{diag}\left(n_{1}^{-1} e_{1}^{-2} I_{n_{1}}, \cdots, n_{k}^{-1} e_{k}^{-2} I_{n_{k}}\right) \varepsilon=o_{p}\left(k^{\frac{1}{2}}\right),
$$

这里 $\Phi=\left(\varphi_{i j}\right)_{n \times n}$. 对于 $(2(1+\delta))^{-1}<\alpha_{1}<\alpha_{2}<\nu /(2 \nu+1)$, 令 $c$ 为常数, 并且

$$
D_{k}=\left\{\varepsilon_{i j}, \varphi_{i j}: \min _{1 \leqslant i \leqslant k} \max _{1 \leqslant j \leqslant n_{i}}\left|\varepsilon_{i j}\right| \geqslant k^{-\alpha_{1}}, \quad \max _{1 \leqslant i \leqslant k, 1 \leqslant j \leqslant n_{i}}\left|\varphi_{i j}\right| \leqslant k^{-\alpha_{2}}\right\} .
$$

在事件 $D_{k}$ 上,

$$
v_{i} \geqslant n_{i}^{-1} \max _{1 \leqslant j \leqslant n_{i}}\left|\varepsilon_{i j}-x_{i j}^{\prime}\left(\hat{\beta}_{w}^{(0)}-\beta\right)-\left(B_{m_{k}}^{\prime}\left(t_{i j}\right) \hat{\alpha}_{w}^{(0)}-g\left(t_{i j}\right)\right)\right|^{2} \geqslant\left(1-k^{\alpha_{1}-\alpha_{2}}\right)^{2} \sum_{j=1}^{n_{i}} \varepsilon_{i j}^{2} \geqslant c e_{i},
$$

因此 $e_{i}^{-2} v_{i}^{-1}\left(v_{i}-e_{i}\right)^{2} \leqslant c\left(v_{i}-e_{i}\right)^{2} e_{i}^{-3}$. 在假定 2.1 下易见

$$
\begin{aligned}
\left(v_{i}-e_{i}\right)^{2} & =n_{i}^{-2}\left[\sum_{j=1}^{n_{i}} \varphi_{i j}^{2}-2 \sum_{j=1}^{n_{i}} \varphi_{i j} \varepsilon_{i j}\right]^{2} \leqslant\left[\sum_{j=1}^{n_{i}} \varphi_{i j}^{2}+2\left(\sum_{j=1}^{n_{i}} \varepsilon_{i j}^{2}\right)^{\frac{1}{2}}\left(\sum_{j=1}^{n_{i}} \varphi_{i j}^{2}\right)^{\frac{1}{2}}\right]^{2} \\
& \leqslant c\left[\max _{1 \leqslant i \leqslant k, 1 \leqslant j \leqslant n_{i}} \varphi_{i j}^{2}+e_{i} \max _{1 \leqslant i \leqslant k, 1 \leqslant j \leqslant n_{i}} \varphi_{i j}\right] .
\end{aligned}
$$

另外, 由于 $\left\|\hat{\beta}_{w}^{(0)}-\beta\right\|=O_{p}\left(k^{-1 / 2}\right)$, 所以结合假定 2.3 及引理 1 和 2 , 下式成立:

$$
\begin{aligned}
& \sup _{0 \leqslant t \leqslant 1}\left|B_{m_{k}}(t) \hat{\alpha}_{w}^{(0)}-g(t)\right| \\
\leqslant & \sup _{0 \leqslant t \leqslant 1}\left|B_{m_{k}}(t)\left(B_{m_{k}}^{\prime} B_{m_{k}}\right)^{-1} B_{m_{k}}^{\prime} \varepsilon\right|+\sup _{0 \leqslant t \leqslant 1}\left|B_{m_{k}}(t)\left(B_{m_{k}}^{\prime} B_{m_{k}}\right)^{-1} B_{m_{k}}^{\prime} g-g(t)\right| \\
& +\sup _{0 \leqslant t \leqslant 1}\left|B_{m_{k}}(t)\left(B_{m_{k}}^{\prime} B_{m_{k}}\right)^{-1} B_{m_{k}}^{\prime} X\left(\beta-\hat{\beta}_{w}^{(0)}\right)\right| \\
= & O\left(m_{k}^{-\nu}\right)+O\left(n^{-1} m_{k}\right)+O_{p}\left[\left(n^{-1} m_{k}\right)^{\frac{1}{2}}\right] .
\end{aligned}
$$

类似于文献 [11] 中引理 2.3 的证明, 有

$$
k^{-5 \alpha} \sum_{i=1}^{k} e_{i}^{-\frac{5}{2}} \rightarrow_{p} 0, \quad k^{-3 \alpha} \sum_{i=1}^{k} e_{i}^{-\frac{3}{2}} \rightarrow_{p} 0 .
$$

因此结合 $\hat{\beta}_{w}^{(0)}$ 和 $B_{m_{k}}^{\prime}(t) \hat{\alpha}_{w}^{(0)}$ 的收玫速度及 (3) 式, 在事件 $D_{k}$ 上,

$$
\left\|X^{\prime} M_{B_{m_{k}}} \operatorname{diag}\left[e_{1}^{-2} v_{1}^{-1}\left(v_{1}-e_{1}\right)^{2} I_{n_{1}}, \cdots, e_{k}^{-2} v_{k}^{-1}\left(v_{k}-e_{k}\right)^{2} I_{n_{k}}\right] \varepsilon\right\|=o_{p}\left(k^{\frac{1}{2}}\right) .
$$

另一方面,

$$
\begin{aligned}
P\left(D_{k}^{c}\right) \leqslant & P\left\{\max _{1 \leqslant i \leqslant k, 1 \leqslant j \leqslant n_{i}}\left|x_{i j}^{\prime}\left(\hat{\beta}_{w}^{(0)}-\beta\right)+B_{m_{k}}^{\prime}\left(t_{i j}\right) \hat{\alpha}_{w}^{(0)}-g\left(t_{i j}\right)\right| \geqslant k^{-\alpha_{2}}\right\} \\
& +\sum_{i=1}^{k} P\left\{\max _{1 \leqslant j \leqslant n_{i}}\left|\varepsilon_{i j}\right| \leqslant k^{-\alpha_{1}}\right\} .
\end{aligned}
$$

由 $\hat{\beta}_{w}^{(0)}$ 和 $B_{m_{k}}^{\prime}(t) \hat{\alpha}_{w}^{(0)}$ 的收玫速度知, 上面方程右边的第 1 项趋于 0 , 第 2 项也趋于 0 , 这是因为

$$
P\left\{\max _{1 \leqslant j \leqslant n_{i}}\left|\varepsilon_{i j}\right| \leqslant k^{-\alpha_{1}}\right\} \leqslant P\left\{e_{i} \leqslant k^{-2 \alpha_{1}}\right\} \leqslant P\left\{e_{i}^{-1} \geqslant k^{2 \alpha_{1}}\right\} \leqslant E e_{i}^{-(1+\delta)} k^{-2 \alpha_{1}(1+\delta)}
$$

和 $2 \alpha_{1}(1+\delta)>1$. 这样证明了 $P\left(D_{k}\right) \rightarrow 1$, 因此 (1) 式成立. 同样能够证明 (2) 式也成立. 由 (1) 和 (2) 式可得

$$
\begin{aligned}
& X^{\prime} M_{B_{m_{k}}} \operatorname{diag}\left(v_{1}^{-1} I_{n_{1}}, \cdots, v_{k}^{-1} I_{n_{k}}\right) \varepsilon \\
= & X^{\prime} M_{B_{m_{k}}} \operatorname{diag}\left(e_{1}^{-1} I_{n_{1}}, \cdots, e_{k}^{-1} I_{n_{k}}\right) \varepsilon+2\left\{\sum_{i=1}^{k} n_{i}^{-1}\left[X_{i}^{\prime}-X^{\prime} B_{m_{k}}\left(B_{m_{k}}^{\prime} B_{m_{k}}\right)^{-1} B_{m_{k} i}\right]\right. \\
& \left.\times \tilde{\varepsilon}_{i} \tilde{\varepsilon}_{i}^{\prime}\left[X_{i}-B_{m_{k} i}\left(B_{m_{k}}^{\prime} B_{m_{k}}\right)^{-1} B_{m_{k}}^{\prime} X\right]\right\}\left(\hat{\beta}_{w}^{(0)}-\beta\right)+o_{p}\left(k^{\frac{1}{2}}\right),
\end{aligned}
$$


其中 $B_{m_{k} i}=\left(B_{m_{k}}\left(t_{i 1}\right), \cdots, B_{m_{k}}\left(t_{i n_{i}}\right)\right)$. 根据假定 2.3 , 下式成立:

$$
\begin{aligned}
& X^{\prime} M_{B_{m_{k}}} \operatorname{diag}\left(e_{1}^{-1} I_{n_{1}}, \cdots, e_{k}^{-1} I_{n_{k}}\right) \varepsilon \\
= & U^{\prime} \operatorname{diag}\left(e_{1}^{-1} I_{n_{1}}, \cdots, e_{k}^{-1} I_{n_{k}}\right) \varepsilon+U^{\prime} P_{B_{m_{k}}} \operatorname{diag}\left(e_{1}^{-1} I_{n_{1}}, \cdots, e_{k}^{-1} I_{n_{k}}\right) \varepsilon \\
& +H^{\prime} M_{B_{m_{k}}} \operatorname{diag}\left(e_{1}^{-1} I_{n_{1}}, \cdots, e_{k}^{-1} I_{n_{k}}\right) \varepsilon,
\end{aligned}
$$

此处 $H=\left(h_{s}\left(t_{i j}\right)\right)_{n \times p}$. 令 $S_{m_{k} l}$ 为 $B_{m_{k} l}(\cdot)$ 的支撑, 则 $S_{m_{k} l}=\left[(l-\nu+1) m_{k}^{-1},(l+\nu+1) m_{k}^{-1} r\right]$. 定义 $M_{m_{k} l}=\left\{i, j: t_{i j} \in S_{m_{k} l}\right\}$ 和 $n_{m_{k} l}$ 等于 $M_{m_{k} l}$ 中 $(i, j)$ 的个数. 明显地对所有 $l$ 和某个 $c>0, n_{m_{k}} l / n \leqslant c m_{k}^{-1}$, 因此结合引理 1 和 2 , 对于 $s=1, \cdots, p$, 有下式成立:

$$
\begin{aligned}
& E\left[\sum_{i_{1}=1}^{k} \sum_{j_{1}=1}^{n_{i_{1}}} e_{i_{1}}^{-1} \varepsilon_{i_{1} j_{1}} \sum_{i_{2}=1}^{k} \sum_{j_{2}=1}^{n_{i_{2}}} u_{i_{2} j_{2} s} B_{m_{k}}^{\prime}\left(t_{i_{2} j_{2}}\right)\left(B_{m_{k}}^{\prime} B_{m_{k}}\right)^{-1} B_{m_{k}}\left(t_{i_{1} j_{1}}\right)\right]^{2} \\
= & O\left(k^{-2} m_{k}^{2}\right) \sum_{(i, j) \in I_{m_{k} l}}\left\{\sum_{l=1}^{m_{k}+\nu} \sum_{\left(i_{2}, j_{2}\right) \in M_{m_{k} l}}\left[B_{m_{k} l}^{2}\left(t_{i_{1} j_{1}}\right)+B_{m_{k} l}^{2}\left(t_{i j}\right)\right]\right\}^{2}=o(k) .
\end{aligned}
$$

另外由引理 2 ,

$$
E\left\{\sum_{i=1}^{k} \sum_{j=1}^{n_{i}} e_{i}^{-1} \varepsilon_{i j}\left[h_{s}\left(t_{i j}\right)-B_{m_{k}}^{\prime}\left(t_{i j}\right)\left(B_{m_{k}}^{\prime} B_{m_{k}}\right)^{-1} h_{s}\right]\right\}^{2}=o(k),
$$

其中 $h_{s}=\left(h_{s}\left(t_{11}\right), \cdots, h_{s}\left(t_{1 n_{1}}\right), \cdots, h_{s}\left(t_{k n_{k}}\right)\right)$, 因此

$$
X^{\prime} M_{B_{m_{k}}} \operatorname{diag}\left(e_{1}^{-1} I_{n_{1}}, \cdots, e_{k}^{-1} I_{n_{k}}\right) \varepsilon=U^{\prime} \operatorname{diag}\left(e_{1}^{-1} I_{n_{1}}, \cdots, e_{k}^{-1} I_{n_{k}}\right) \varepsilon+o_{p}\left(k^{\frac{1}{2}}\right) .
$$

同样地能够证明

$$
\begin{aligned}
& \left\{\sum_{i=1}^{k} n_{i}^{-1}\left[X_{i}^{\prime}-X^{\prime} B_{m_{k}}\left(B_{m_{k}}^{\prime} B_{m_{k}}\right)^{-1} B_{m_{k} i}\right] \tilde{\varepsilon}_{i} \tilde{\varepsilon}_{i}^{\prime}\left[X_{i}-B_{m_{k} i}\left(B_{m_{k}}^{\prime} B_{m_{k}}\right)^{-1} B_{m_{k}}^{\prime} X\right]\right\}\left(\hat{\beta}_{w}^{(0)}-\beta\right) \\
= & {\left[\sum_{i=1}^{k} n_{i}^{-1} U_{i}^{\prime} \tilde{\varepsilon}_{i} \tilde{\varepsilon}_{i}^{\prime} U_{i}\right]\left(\hat{\beta}_{w}^{(0)}-\beta\right)+o_{p}\left(k^{\frac{1}{2}}\right) . }
\end{aligned}
$$

综上所述有

$$
\begin{aligned}
& X^{\prime} M_{B_{m_{k}}} \operatorname{diag}\left(v_{1}^{-1} I_{n_{1}}, \cdots, v_{k}^{-1} I_{n_{k}}\right) \varepsilon \\
= & U^{\prime} \operatorname{diag}\left(e_{1}^{-1} I_{n_{1}}, \cdots, e_{k}^{-1} I_{n_{k}}\right) \varepsilon+2\left[\sum_{i=1}^{k} n_{i}^{-1} U_{i}^{\prime} \tilde{\varepsilon}_{i} \tilde{\varepsilon}_{i}^{\prime} U_{i}\right]\left(\hat{\beta}_{w}^{(0)}-\beta\right)+o_{p}\left(k^{\frac{1}{2}}\right) .
\end{aligned}
$$

令 $M_{B_{m_{k}}} X=\left(\hat{x}_{i j s}\right)_{n \times p}$, 则 $X^{\prime} M_{B_{m_{k}}} \operatorname{diag}\left(v_{1}^{-1} I_{n_{1}}, \cdots, v_{k}^{-1} I_{n_{k}}\right) P_{B_{m_{k}}} \varepsilon$ 的第 $s$ 个元素是

$$
\begin{aligned}
& \sum_{i=1}^{k}\left[e_{i}^{-1}+2 n_{i}^{-1} e_{i}^{-2} \sum_{j=1}^{n_{i}} \varphi_{i j} \varepsilon_{i j}+n_{i}^{-1} e_{i}^{-2} \sum_{j=1}^{n_{i}} \varphi_{i j}^{2}+e_{i}^{-2} v_{i}^{-1}\left(v_{i}-e_{i}\right)^{2}\right] \\
& \cdot \sum_{h=1}^{n_{i}} \hat{x}_{i h s} B_{m_{k}}^{\prime}\left(t_{i h}\right)\left(B_{m_{k}}^{\prime} B_{m_{k}}\right)^{-1} B_{m_{k}}^{\prime} \varepsilon \hat{=} J_{1}+\cdots+J_{4}
\end{aligned}
$$

类似于 $U^{\prime} P_{B_{m_{k}}} \operatorname{diag}\left(e_{1}^{-1} I_{n_{1}}, \cdots, e_{k}^{-1} I_{n_{k}}\right) \varepsilon=o_{p}\left(k^{1 / 2}\right)$ 的证明, 可得到 $J_{1}=o_{p}\left(k^{1 / 2}\right)$. 根据 You 和 Chen 文 1) 中引理 2.5 的证明, 有 $J_{i}=o_{p}\left(k^{1 / 2}\right)(i=2,3)$. 类似于 (1) 式的证明, 可得到 $J_{4}=o_{p}\left(k^{1 / 2}\right)$, 因此 $X^{\prime} M_{B_{m_{k}}} \operatorname{diag}\left(v_{1}^{-1} I_{n_{1}}, \cdots, v_{k}^{-1} I_{n_{k}}\right) P_{B_{m_{k}}} \varepsilon=o_{p}\left(k^{1 / 2}\right)$.

1) You J, Chen G. Two-stage estimation for a partly linear regression model with unknown diagonal covariance matrix. Technical Report, Department of Mathematics \& Statistics, University of Regina. 2000 
因为 $X^{\prime} M_{B_{m_{k}}} \operatorname{diag}\left(v_{1}^{-1} I_{n_{1}}, \cdots, v_{k}^{-1} I_{n_{k}}\right) M_{B_{m_{k}}} g$ 的第 $s$ 个元素可分解为

$$
\begin{aligned}
& \sum_{i=1}^{k}\left[e_{i}^{-1}+2 n_{i}^{-1} e_{i}^{-2} \sum_{j=1}^{n_{i}} \varphi_{i j} \varepsilon_{i j}+n_{i}^{-1} e_{i}^{-2} \sum_{j=1}^{n_{i}} \varphi_{i j}^{2}+e_{i}^{-2} v_{i}^{-1}\left(v_{i}-e_{i}\right)^{2}\right] \\
& \cdot \sum_{h=1}^{n_{i}} \hat{x}_{i h s}\left[g\left(t_{i h}\right)-B_{m_{k}}\left(t_{i h}\right)\left(B_{m_{k}}^{\prime} B_{m_{k}}\right)^{-1} B_{m_{k}}^{\prime} g\right] \hat{=} K_{1}+\cdots+K_{4},
\end{aligned}
$$

由文献 [11] 中的引理 $2.1, K_{1}$ 依概率收敛到

$$
\sum_{i=1}^{k} \sum_{j=1}^{n_{i}} \hat{x}_{i j s} n_{i} \tau\left(n_{i}\right) \sigma_{i}^{-2}\left[g\left(t_{i j}\right)-B_{m_{k}}\left(t_{i j}\right)\left(B_{m_{k}}^{\prime} B_{m_{k}}\right)^{-1} B_{m_{k}}^{\prime} g\right]
$$

其中 $\tau\left(n_{i}\right)=\sigma_{i}^{2} E\left(\sum_{j=1}^{n_{i}} \varepsilon_{i j}^{2}\right)^{-1}$. 根据假定 2.3 和 Abel 不等式, $|(4)|=o\left(k^{1 / 2}\right)$ 成立. 结合引理 1 , 类似于 $J_{2}, J_{3}$ 和 $J_{4}$ 的证明, 可以得到 $K_{i}=o_{p}\left(k^{1 / 2}\right)(i=2,3,4)$, 因此

$$
X^{\prime} M_{B_{m_{k}}} \operatorname{diag}\left(v_{1}^{-1} I_{n_{1}}, \cdots, v_{k}^{-1} I_{n_{k}}\right) M_{B_{m_{k}}} g=o_{p}\left(k^{\frac{1}{2}}\right) .
$$

类似于 (1) 式的证明, 下式成立:

$$
k\left\{\left[X^{\prime} M_{B_{m_{k}}} \operatorname{diag}\left(v_{1}^{-1} I_{n_{1}}, \cdots, v_{k}^{-1} I_{n_{k}}\right) M_{B_{m_{k}}} X\right]-\widehat{G}_{k}^{-1}\right\} \rightarrow_{p} 0,
$$

其中 $\widehat{G}_{k}=X^{\prime} M_{B_{m_{k}}} \operatorname{diag}\left(E e_{1}^{-1} I_{n_{1}}, \cdots, E e_{k}^{-1} I_{n_{k}}\right) M_{B_{m_{k}}} X$. 进一步地, 由引理 1 和 2 易见 $k\left(\widehat{G}_{k}^{-1}-G^{-1}\right) \rightarrow 0$, 因此

$$
k\left\{\left[X^{\prime} M_{B_{m_{k}}} \operatorname{diag}\left(v_{1}^{-1} I_{n_{1}}, \cdots, v_{k}^{-1} I_{n_{k}}\right) M_{B_{m_{k}}} X\right]-G_{k}^{-1}\right\} \rightarrow_{p} 0 .
$$

由相同的方法可以证明

$$
\frac{1}{k}\left[\sum_{i=1}^{k} \frac{1}{n_{i}}\left(X_{i}^{\prime}-X B_{m_{k}}\left(B_{m_{k}}^{\prime} B_{m_{k}}\right)^{-1} B_{m_{k} i}\right) \tilde{\varepsilon}_{i} \tilde{\varepsilon}_{i}^{\prime}\left(X_{i}-B_{m_{k}}\left(B_{m_{k}}^{\prime} B_{m_{k}}\right)^{-1} B_{m_{k}}^{\prime} X^{\prime}\right)-H_{k}\right] \rightarrow_{p} 0 .
$$

证毕.

定理 2.1 的证 令 $\left\{A_{k}^{(0)}\right\}$ 和 $\left\{C_{k}^{(0)}\right\}$ 为两个 $p \times\left(\sum_{i=1}^{k} n_{i}\right)$ 的矩阵序列, 它们满足 $\left(A_{k}^{(0)}\right)\left(A_{k}^{(0)}\right)^{\prime}=O(k)$ 和 $\left(C_{k}^{(0)}\right)\left(C_{k}^{(0)}\right)^{\prime}=O(k)$. 对于任意的正整数 $s$, 令

$$
A_{k}^{(s)}=\sum_{l=0}^{s-1}\left(2 H_{k} G_{k}^{-1}\right)^{l} U^{\prime}+\left(2 H_{k} G_{k}^{-1}\right)^{s} A_{k}^{(0)}, \quad C_{k}^{(s)}=\left(2 H_{k} G_{k}^{-1}\right)^{s} C_{k}^{(0)},
$$

其中 $G_{k}$ 和 $H_{k}$ 见引理 3. 令 $A_{k}=A_{k}^{(l-1)}, C_{k}=C_{k}^{(l-1)}, \hat{\beta}_{w}^{(0)}=\hat{\beta}_{w}^{(l-1)}$ 和 $\hat{\beta}_{w}^{(1)}=\hat{\beta}_{w}^{(l)}(l=1,2, \cdots, s)$, 重复使用 $\delta$ 方法及引理 3 , 可以得到定理 2.1 . 